\title{
Ear and Labyrinth Disorder Class
}

National Cancer Institute

\section{Source}

National Cancer Institute. Ear and Labyrinth Disorder Class. NCI Thesaurus. Code C49400.

Any deviation from the normal structure or function of the ear or labyrinth that is manifested by a characteristic set of symptoms and signs. 